THE AUTHOR FILE

\section{Abbas El Gamal and Mark Schnitzer}

Two-gram microscopes make brain images in moving mice.

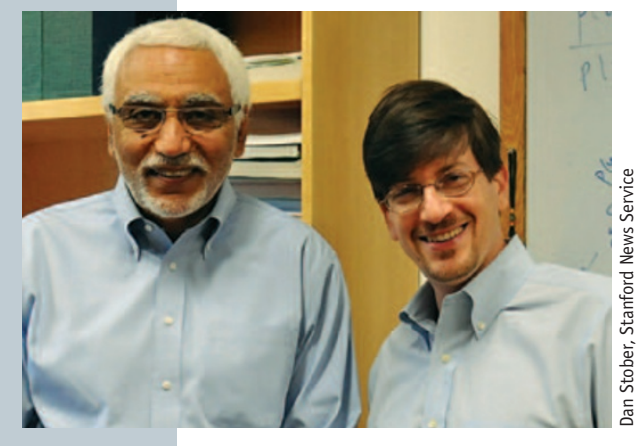

Abbas El Gamal and Mark Schnitzer a graduate student in Abbas El Gamal's laboratory, wanted to make a sensor better suited for biological imaging. Across Stanford University's Campus Drive, his friend, Eric Tatt Wei Ho, a graduate student in Mark Schnitzer's lab, was working on ways of imaging neural activity. In a chance conversation, the two wondered whether there was a way to put an imaging sensor directly onto an animal.

Ghosh and El Gamal approached Schnitzer with the idea. As it happened, Schnitzer had played with the concept before while working at Bell Labs. Back then, though, neither electronic devices nor imaging technology were advanced enough to make such a project feasible. At Stanford University, Schnitzer had designed miniature fluorescence microscopes that could peer into mouse brains by using optical fibers to carry light and signals in and out of the brain, but he was looking for better technologies. "This proposal hit us at the right time," says Schnitzer. Laurie Burns, Eric Cocker and other members of the Schnitzer lab joined the project.

The collaborators quickly decided not to go with the original plan of designing a new imaging sensor. "Instead we designed a whole system," says El Gamal. The resulting 1.9-gram microscope consists of a semiconductor image sensor, a light-emitting diode (LED), a focusing mechanism, a mirror, lenses and a filter, plus nine wires to carry power and information. The field of view and amount of data collected are improved several-fold over previous systems, allowing the microscope to resolve changes in blood flow in cerebellar capillaries on the scale of seconds and to track calcium spiking in over 200 individual neurons concurrently.

One reason for the improvements is that the light comes from the head-mounted LED rather than being piped in through fiber optics. In previous systems, irregularities from the light source would change orientation as the animal itself moved, creating distortions that had to be filtered out. Having a light source that actually moves with the mouse greatly improved the quality of the data, says Schnitzer. The flexible wires also let mice move more freely than more rigid fibers do. "We're not using the prior fiber optic microscope anymore," he says.

Meanwhile Ghosh has started a company, Inscopix, to commercialize the microscopes. (Cocker, El Gamal and Schnitzer also have equity in the company.) These small, mass-producible microscopes could have many uses, says Schnitzer. Individual microscopes can even be integrated into arrays. Experiments published in this issue of Nature Methods demonstrate monitoring four zebrafish brains simultaneously and quickly counting cells in 96-well plates.

The optoelectronic components of the microscope are all already mass-produced, but getting them to work together in a minifluorescence microscope was not straightforward. The team set up an integrated simulation system, which allowed them to tweak parameters and specifications in a computer model. This allowed them to test, for example, how a particular sensor would respond to different types of photon flux. They went through a lot of design candidates, says Schnitzer. "We spent about a year in silico." Otherwise, the project wouldn't have been possible, says El Gamal. "We couldn't afford to just slap these things together."

The investment paid off: the microscope worked exactly as designed the very first time they mounted it onto a mouse's head. It is also physically robust enough to sustain the abuse that a mouse delivers, such as bonking its head against an enclosure or scratching behind its ear.

Tourists' desire to snap pictures with their cell phones has prompted millions of dollars worth of technology investments, and that has brought unanticipated benefits, says Schnitzer. "The telecom market is so huge that it has propelled these sensors to an exquisite level. Despite the fact that they never had mouse brain imaging in mind, we can harvest the fruit of their investments. You can just assemble parts that have already been proven rather than fabricating something new."

The collaboration, reflects El Gamal, is very different from a typical engineering project to design a new kind of chip. In this case the point is not engineering some new proof of concept but in making it function in the real world. "The benefit is not in the device itself but demonstrating that it works in biology," he says.

Monya Baker

Ghosh, K.K. et al. Miniaturized integration of a fluorescence microscope. Nat. Methods 8, 871-878 (2011). 